\title{
Clinical Implication of Targeting of Cancer Stem Cells
}

\author{
L. Zhao Y.Zhao Q. Bao H. Niess K.-W. Jauch C.J.Bruns \\ Department of Surgery, Munich Medical Center, Campus Grosshadern, LMU Munich, Munich, Germany
}

\section{Key Words}

Cancer stem cells $\cdot$ Target therapy $\cdot$ Resistance $\cdot$ Signaling pathway $\cdot$ Molecular markers

\begin{abstract}
The existence of cancer stem cells (CSCs) is receiving increasing interest particularly due to its potential ability to enter clinical routine. Rapid advances in the CSC field have provided evidence for the development of more reliable anticancer therapies in the future. CSCs typically only constitute a small fraction of the total tumor burden; however, they harbor self-renewal capacity and appear to be relatively resistant to conventional therapies. Recent therapeutic approaches aim to eliminate or differentiate CSCs or to disrupt the niches in which they reside. Better understanding of the biological characteristics of CSCs as well as improved preclinical and clinical trials targeting CSCs may revolutionize the treatment of many cancers. Copyright $\odot 2012 \mathrm{~S}$. Karger AG, Basel
\end{abstract}

\section{Introduction}

During the past decades, researchers keenly endeavored to develop effective anticancer therapies. However, the incidence and the mortality of cancers are still in- creasing despite persistently ongoing research. Despite conventional therapies such as surgical resection, radiotherapy and chemotherapy, most patients suffer from cancer recurrence. As the malignant tumor is constituted by a morphologically and functionally heterogeneous population of cells, and the majority of cells are destined to differentiate or transit-amplify, a minor cell population is defined as a group of undifferentiated cells with the ability (i) to duplicate themselves and self-renew, (ii) to regenerate a phenocopy of the original tumor and drive neoplastic proliferation, when transplanted into the nonobese diabetic/severe combined immunodeficient mouse, and (iii) to differentiate to some degree into more mature non-stem-cell cancer lineages. These cells referred to as cancer stem cells (CSCs) received increasing attention as novel targets for cancer therapy, in particular as emerging evidence indicates that CSCs are substantially associated with tumor initiation, angiogenesis, cancer maintenance and metastasis [1,2]. Actually, the clinically most relevant cancer cells are probably not those that engraft immunodeficient mice, but rather those that are responsible for cancer relapse. Accordingly, even if every cancer cell possesses tumorigenic potential, the more clinically relevant question is probably whether any cell can also be responsible for relapse or only a discrete cell subset [3]. Even more challenging is the established tight interaction between a tumor and its environment [4], which can trans-

\section{KARGER}

Fax +4161306 1234 E-Mail karger@karger.ch www.karger.com
(C) 2012 S. Karger AG, Basel

0014-312X/12/0491-0008\$38.00/0

Accessible online at:

www.karger.com/esr
Lu Zhao

Department of Surgery, Munich Medical Center, Campus Grosshadern,

Ludwige Maximillian University, Marchioninistraße 15

DE-81377 Munich (Germany)

Tel. +49 897095 3431, E-Mail Lu.Zhao@med.uni-muenchen.de 
fer nonstem cells into CSCs, since the CSC phenotype is more alternating than anticipated and strongly regulated by the tumor cell environment [5]. In any case, of greatest clinical implication is the development of an effective treatment regimen that prevents survival, self-renewal and differentiation of CSCs and disturbs the microenvironmental niche of CSCs without damaging normal stem cells. In this premise, we highlight the latest clinical developments in CSCs targeting therapy and discuss the stumbling blocks which stand on the way to our expected target therapy goal.

\section{The Dandelion Phenomenon}

The therapy of chronic myeloid leukemia (CML) has evolved significantly over the past two decades. Before the availability of imatinib, $\alpha$-interferon was used in high doses and was associated with the achievement of hematological and even molecular remission in individuals with CML; however, significant toxicities were frequently encountered including depression and neurological disturbances. Imatinib was found to be superior for achieving cytogenetic and molecular responses and prolonging event-free survival as compared to $\alpha$-interferon $[6,7]$. However, the long-term follow-up of participants continues to show that not all patients remain in longterm molecular remission with imatinib therapy and that as many as $25-30 \%$ of the patients become resistant $[7,8]$. CML patients who achieve the best responses to imatinib can relapse quickly when the drug is discontinued [9-12] or even progress while remaining on the drug [12].

This phenomenon where a therapy eliminates most of the tumor bulk but spares the CSCs could be considered analogous to mowing dandelions; although this will eliminate the visible portion of the weeds, the unseen roots also need to be addressed to prevent regrowth [3, 13-15]. In contrast, a treatment addressing CSCs would be comparable to attacking just the root of the dandelion; although this therapy has no immediate discernible effect on the weeds, over time the weeds should eventually wither and die when the roots have been eliminated [3, 13-15].

The dandelion phenomenon can be applied in many studies, since the achievement of a complete response and total eradication of all macroscopic diseases has not been associated with increased overall survival as compared to patients achieving only partial remission following treatment. For example, several large randomized studies using combination chemotherapy in pancreatic adenocarcinoma have demonstrated improved rates of tumor re- sponse but only negligible or no improvements in overall survival [16]. CSCs might be accountable for this discrepancy in many malignancies as tumor response primarily reflects short-term changes regarding the bulk of tumor cells, whereas long-term outcome represented by disease relapse based on local or systemic progression and overall survival might be attributed to CSCs [16].

\section{Clinical Relevance of CSCs}

Due to the common property of CSCs including selfrenewal capacity, tumorigenicity, proliferative capacity and resistance to standard antitumor therapy, it is obvious to posit that metastasis formation as well as cancer relapse are closely associated with CSCs [17].

A number of recent researches have examined whether CSCs can serve as predictive biomarkers and have included studies examining the association between the frequency of CSCs, the expression of CSC-specific gene signatures and the qualification of functional CSC properties with clinical outcome $[16,18]$. In human acute myeloid leukemia, a link between leukemic stem cell (LSC) burden and clinical behavior by showing that undifferentiated acute myeloid leukemia (FAB subtype M0) has a higher frequency of LSCs and a poorer prognosis than other FAB subtypes have suggested [18-20]. A more recent study directly addressed the relationship between LSC frequency and clinical outcome, demonstrating that the frequency of LSCs at the time of diagnosis correlates with several clinical endpoints, including overall survival, disease-free and relapse-free survival [18].

CSCs also correlate with the prognosis of gliomas. A recent study found high levels of the neural progenitor cell marker nestin in more aggressive high-grade gliomas. High-grade gliomas also coexpressed high levels of cysteine [21] and cathepsins B and L, two further markers for invasion. A coexpression of both markers indicated a poorer prognosis and a significantly shorter survival of glioma patients [22]. By Cox regression analysis, nestin was shown to be a stronger prognostic marker than cathepsins B and L [21]. In addition, in breast cancer the prognostic value of CSC markers in the clinic has begun to emerge. Tumors mainly composed of CD44+CD24PROCR+ cells presented with a worse clinical outcome than tumors with a CD44-CD24+ signature [23]. Recently, Liu et al. [24] compared a 186-gene-containing expression profile of CD44+CD24-/low putative breast CSCs to that of normal breast epithelium and found a statistically significant association between the gene sig- 
nature in both overall and metastasis-free survival in addition to established clinical and pathological variables. This unique gene signature was then used to stratify patients into high-risk (10-year survival of $81 \%$ ) and lowrisk (10-year survival of 57\%) patients in combination with prognostic criteria of the National Institutes of Health. The gene signature was further associated with the prognosis in medulloblastoma, lung cancer and prostate cancer $[22,24]$. In summary, the genetic profile of CSCs may serve as useful predictor for clinical outcome.

\section{CSCs Are Resistant to Conventional Anticancer Therapy}

Current anticancer therapy strategies mainly include chemotherapy, radiotherapy and surgery; so far these therapeutic strategies either alone or in combination fail to eradicate CSC clones and instead favor the expansion of the CSC pool and/or select for resistant CSC clones, thus leading to a fatal outcome of the disease [23].

\section{Chemotherapy}

Most chemotherapies eradicate a substantial part of the tumor cells; however, CSCs which stay dormant for long periods during therapy will eventually reenter the cell cycle and recapitulate tumor regrowth since their ability of chemoresistance is increasing under chemotherapy [25]. There is quite a lot of evidence for enhanced chemoresistance of CSCs: Liu et al. [26] first demonstrated that CD133+ brain tumor stem cells disclosed a survival advantage to chemotherapeutic agents such as temozolomide, carboplatin, paclitaxel and etoposide compared to autologous CD133- cells [25]. CD133 has proven to be a marker for neuroepithelial, hematopoietic, breast and endothelial progenitor cells [23, 27-29]. Furthermore, studies in patients with CML showed that CML stem cells are resistant to imatinib treatment independently from the existence of resistance-associated mutations in the BCR-ABL gene [5, 30, 31]. Similarly, in gastrointestinal stromal tumors CSCs seem to be resistant to imatinib [32]. In addition, in colorectal cancer after conventional chemotherapy with oxaliplatin, irinotecan and fluorouracil, the CSC population is enlarged and more resistant to chemotherapy than non-CSCs in xenograft mouse models [5, 33-35]. Comparably, Szotek et al. [36] showed that CSCs of ovarian cancer could not be inhibited by doxorubicin unlike non-CSCs.

There are several properties that contribute to the chemoresistance of CSCs as shown below.

\section{Quiescent Phenotype}

Conventional chemotherapies largely rely on cycling cells in order to cause lethal cellular damage; in contrast, CSCs stay dormant during or after the treatment for a long time until reentry of the cell cycle to stimulate tumor cell proliferation [1].

\section{Antiapoptosis}

Based on the expression of antiapoptotic proteins such as BCL-2 some self-renewal pathways such as transforming growth factor $\beta$, Wnt $/ \beta$-catenin or BMI-1 are activated and lead to enhanced capability to repair DNA damage after genotoxic stress or activation of autocrine loops through the production of growth factors like epidermal growth factor [37].

Expression of Certain Drug Efflux Pumps

Typical drug efflux proteins such as ABCC1, ABCG2 or MDR1, which are the principal mediators of multidrug resistance identified so far, are expressed on CSCs [37]. Multidrug resistance-associated proteins (ABCC subfamily) are members of the ATP-binding cassette (ABC) superfamily of transport proteins and act as cellular efflux transporters for a wide variety of substrates, in particular glutathione, glucuronide and sulfate conjugates of diverse compounds. Together with P-glycoprotein (MDR1; ABCB1) and breast cancer resistance protein (ABCG2), the multidrug resistance-associated proteins are highly important as cellular defense against toxicants and confer multixenobiotic resistance [38]. In the breast cancer cell lines MCF-7 and Cal-51, recently side population cells expressing ABCG2 transporter properties have been isolated. Purified MCF-7 side population cells further demonstrated an increased ability to colonize the mouse mammary gland as compared to non-side-population MCF-7 cells [4]. In metastatic human pancreatic cancer cells (L3.6pl), side population cells have been identified as potential CSCs being responsible for gemcitabine resistance [39]. These cells also displayed the capacity of self-renewal, differentiation, induction of aggressive tumor growth, as well as high rates of metastasis [unpubl. data]. In summary, the resistance properties of CSCs analyzed above provide some potential targets for anti-CSC therapy.

\section{Radiotherapy}

Recent investigations on CSC-associated resistance to radiotherapy are mainly related to breast cancer and glioblastoma multiforme. In glioblastoma multiforme, scientists showed that CD133+ CSCs contributed to glioma ra- 
dioresistance through preferential activation of the highly efficient DNA damage repair pathway, less apoptosis by significantly induced checkpoint kinases and an increase in DNA repair capacity compared to CD133-tumor cells $[25,40]$. In breast cancer, progenitor cells in the mammary gland are more resistant to clinically relevant doses of radiation than nonprogenitor cells [41]. Diehn et al. [42] demonstrated that reduced levels of reactive oxygen species are associated with radioresistance in CSCs. In addition, Chen et al. [43] identified that the Wnt/ $\beta$ catenin pathway mediates radiation resistance of progenitor cells in an immortalized mammary gland cell line.

Moreover, the tumor microenvironment is substantially involved in the distribution of CSCs within the tumour mass and the mediation of the radiotherapy response. Recent research reports identified hypoxia as crucial for the expansion and maintenance of CSCs in different tumor entities [37, 44,45]. Selective therapy of hypoxic areas within the tumor would eradicate CSC niches. Modern radiotherapy techniques might allow to accurately deliver selective doses of radiotherapy to hypoxic areas of the tumor instead of an inhomogeneous dose distribution within the tumor mass $[37,46]$.

\section{Surgery}

Although surgery is frequently still the gold standard of curative treatment and has a broad indication for many solid neoplasms, most patients ultimately suffer from local recurrence or systemic relapse due to metastasis based on minimal residual disease which is enriched for CSCs. One example is glioblastoma multiforme, the most malignant and aggressive type of brain tumor. At present, even the combination of surgical resection, advanced chemotherapy as well as radiotherapy remains a palliative therapy with an average recurrence-free survival of only 6.9 months and a 5 -year survival rate of less than $5 \%[1,47]$.

\section{How to Target CSCs More Accurately?}

Based on the previous knowledge of the biological character of CSCs one can conclude that CSC targeting therapy (table 1) should be designed on the following principles: eliminating CSCs by either killing or differentiating them, and/or disrupting the niches and microenvironments in which the CSCs reside [17]. Efficient eradication of CSCs may require the combined ablation of CSCs themselves and their niches. Regarding preferential microenvironments of CSCs, controversial reports exist: there are perivascular as well as hypoxic niches [17].

Clinical Implication of Targeting of Cancer Stem Cells

\section{Identification of CSC Markers}

CSCs have been isolated and purified from cancers of the breast, brain, thyroid, cervix, lung, blood (leukemia), skin (melanoma), organs of the gastrointestinal and reproductive tracts, and the retina using cell surface molecules as markers [48].

Since CSCs share the same or similar cell markers with normal stem cells, CSC-targeted therapy will eventually damage normal stem cells during the treatment, so it is substantial to identify more specific CSC markers.

More recently, advanced techniques such as signal sequence trap (SST) PCR screening methods have been developed to identify a leukemia-specific stem cell marker (CD96) [49]. The SST PCR screening method possessed the ability to redirect a constitutively active mutant of a cytokine receptor to the cell membrane detecting mRNAs that contain signal sequences on cDNA fragments and thereby inducing interleukin-3-independent growth of a mouse B cell line Ba/F3 [50]. Hosen et al. [49] identified with SST PCR CD96 which was much more highly expressed in different CD34+CD38- acute myeloid leukemia blasts. The implication is that CSCs can be primarily enriched using currently identified surface molecules in conjunction with a subsequent approach such as SST that can be assembled for additional marker identification [22].

It has already been shown that proteins such as nodal and activin are expressed during embryonic development of the mesoderm formation and the left-right axis specification [51]. Recently, researchers found that knockdown or pharmacological inhibition of the nodal/activin receptor Alk4/7 in CD133+/CD44+ pancreatic CSCs virtually abrogated their self-renewal capacity as well as in vivo tumorigenicity, and reversed the resistance of orthotopically engrafted CSCs to gemcitabine. The additional application of a stroma-targeting hedgehog pathway inhibitor enhanced the delivery of the nodal/activin inhibitor and resulted in long-term progression-free survival of the animals. Therefore, inhibition of the Alk4/7 pathway, if combined with inhibition of the hedgehog pathway and gemcitabine, provides a therapeutic strategy for targeting CD133+/CD44+ pancreatic CSCs [51]. Further identification of new CSC surface markers may provide additional targets for the design of anti-CSC therapy [52].

\section{Targeting Specific Molecular Pathways in CSCs}

Normal and malignant stem cells share important functional pathways; thus, it is essential to develop CSCselective therapies without potential side effects addressing normal stem cell function. Those pathways include 
Table 1. Selection of methods used for CSC-targeting therapy

\begin{tabular}{|c|c|c|c|}
\hline Target type & Specific target & Characteristics & Ref. \\
\hline \multirow{7}{*}{$\begin{array}{l}\text { Cell } \\
\text { markers }\end{array}$} & $\mathrm{CD} 24+\mathrm{CD} 44+\mathrm{ESA}+$ & Pancreatic CSCs, expression level is elevated during tumorigenesis & 25,72 \\
\hline & CD44+CD24-ESA+ & Breast CSCs & 73 \\
\hline & EpCAM ${ }^{\text {high }} \mathrm{CD} 44+\mathrm{CD} 166+$ & Colorectal cancer & 74 \\
\hline & CD34+CD38- & AML, broad use as a target for chemotherapy & 75 \\
\hline & CD133+ & $\begin{array}{l}\text { Prostate and breast CSCs, } 5 \text {-transmembrane domain cell surface glycopro- } \\
\text { tein, also a marker for neuron epithelial, hematopoietic and endothelial } \\
\text { progenitor cells }\end{array}$ & 1,28 \\
\hline & Stro1+CD105+CD44+ & A surface marker for bone sarcoma & 76 \\
\hline & Nodal/activin & $\begin{array}{l}\text { Knockdown or pharmacological inhibition of the nodal/activin receptor } \\
\text { Alk } 4 / 7 \text { in CSCs virtually abrogated their self-renewal capacity and in vivo } \\
\text { tumorigenicity }\end{array}$ & 51 \\
\hline \multirow[t]{4}{*}{$\begin{array}{l}\text { Signaling } \\
\text { pathways }\end{array}$} & Hedgehog & $\begin{array}{l}\text { Being upregulated in many cancer types, inhibitors: GDC-0449, } \\
\text { PF04449913, BMS-833923, IPI-926 and TAK-441 }\end{array}$ & $1,16,75$ \\
\hline & $\mathrm{Wnt} / \beta$-catenin & $\begin{array}{l}\text { Be required for CSC self-renewal and tumor growth in different cancers, } \\
\text { including CML and squamous cell carcinoma, inhibitors: PRI-724, WIF-1 } \\
\text { and telomerase }\end{array}$ & $1,16,22$ \\
\hline & Notch & $\begin{array}{l}\text { An important regulator in normal development, adult stem cell mainte- } \\
\text { nance, and tumorigenesis in multiple organs, inhibitors: RO4929097, } \\
\text { BMS-906024, IPI-926 and MK0752 }\end{array}$ & 1,16 \\
\hline & PI3K/Akt/PTEN/mTOR & $\begin{array}{l}\text { Is deregulated in many tumors and used to preferentially target CSCs, in- } \\
\text { hibitors: temsirolimus, everolimus FDA-approved therapy for renal cell } \\
\text { carcinoma }\end{array}$ & 75 \\
\hline \multirow[t]{2}{*}{ Niche } & Angiogenesis (VEGF) & $\begin{array}{l}\text { Inhibitor: bevacizumab results in a disruption of the CSC niche, depleted } \\
\text { vasculature and a dramatic reduction in the number of CSCs in colon, } \\
\text { breast and non-small-cell lung cancer }\end{array}$ & 61 \\
\hline & Hypoxia (HIF pathway) & $\begin{array}{l}\text { Inhibitors: topotecan and digoxin have been approved to be clinically ap- } \\
\text { plied in ovarian, lung and cervical cancer }\end{array}$ & 17 \\
\hline \multirow[t]{4}{*}{ miRNAs } & miR-200 family & $\begin{array}{l}\text { The miR-200 family inhibits EMT and cancer cell migration by direct tar- } \\
\text { geting of E-cadherin transcriptional repressors ZEB1 and ZEB2 }\end{array}$ & 64 \\
\hline & Let-7 family & Regulates BT-IC stem cell-like properties by silencing more than one target & 77 \\
\hline & miR-124 & Targets laminin $\gamma_{1}$ and integrin $\beta_{1}$; related to neuronal differentiation & 66 \\
\hline & $\operatorname{miR}-21$ & Suppresses the self-renewal of embryonic stem cells & 78 \\
\hline
\end{tabular}

$\mathrm{AML}=$ Acute myeloid leukemia; FDA $=$ Food and Drug Administration; VEGF $=$ vascular endothelial growth factor; HIF $=$ hypoxia-inducible factor; EMT = epithelial-mesenchymal transition; BT-IC = breast tumor-initiating cell.

Wnt/ $\beta$-catenin, hedgehog, notch, Hox family members, BMI-1, PTEN and nodal/activin [18, 53-55]. For example, cyclopamine as an antagonist of the hedgehog coreceptor SMO significantly eliminated CSCs in CML and impaired the growth of imatinib-resistant mouse and human CML cells [56]. In medulloblastoma, the inhibition of notch signaling through $\gamma$-secretase inhibitors substantially reduced the CD133+ brain CSC population [57]. Meanwhile, inhibition of notch using small molecule inhibitors of $\gamma$-secretase is currently evaluated in phase I and II clinical trials for glioblastoma [58].
The difference between CSCs and normal stem cells regarding telomere length and telomerase activity may provide another therapeutic option against CSCs. Normal stem cells require telomerase to prevent telomere shortening leading to replicative senescence. However, even in the absence of telomerase, normal stem cells can maintain their replicative capacity for some period of time because of their relatively long telomeres. In contrast, constitutive telomerase activity is absolutely required for the maintenance and growth of most malignancies in order to stabilize short telomeres typically for 
CSCs $[3,15,59]$. Thus, the difference in telomere length between normal (long) and cancer (short) stem cells could provide therapeutic selectivity [15].

In a phase II clinical trial, telomerase peptide vaccination using the telomerase peptide GV1001 after chemoradiotherapy of non-small-cell lung cancer proved to increase the disease-free survival and was well tolerated. The majority of non-small-cell lung cancer patients were immunized and established a durable T-cell memory [60].

\section{Targeting CSC Survival Niches}

Targeting the CSC survival niche may disconnect intrinsic and extrinsic signals that maintain and govern CSCs in their division and differentiation. Both epithelial-mesenchymal transition and tumor angiogenesis contribute to microenvironments supporting enrichment of CSCs. Interestingly, CSCs in solid tumors are indeed concentrated around blood vessels depending in part on signals from endothelial cells [17]. Therefore, antiangiogenic therapies such as bevacizumab (antivascular endothelial growth factor antibody) against metastatic colorectal cancer [61] can be considered as a potential strategy to deplete the tumor from CSCs by compromising their niche.

Hypoxia which has also been identified as CSC niche can drive tumor progression by triggering a set of adaptive transcriptional responses that regulate tumor angiogenesis, metabolism, motility and survival [62]. Interestingly, direct or indirect targeting of hypoxia-inducible factors have proven to reduce the CSC-mediated tumor growth [63].

\section{Manipulation of MicroRNA Expression}

MicroRNAs (miRNAs) are small noncoding RNA molecules that can regulate gene expression by interacting with multiple mRNAs inducing either suppression of translation or degradation of mRNA [64]. miRNAs are often deregulated in various cancers and therefore offer therapeutic potential.

In particular, epithelial mesenchymal transition is regulated by signals originating from the tumor microenvironment which in turn modulate miRNA networks in CSC populations [64]. The existence of multiple stem cell states suggests the necessity of developing therapeutic strategies capable of effectively targeting CSCs in all these different states [65]. Since the CSC states are regulated by miRNAs, these small noncoding RNAs may be useful therapeutic agents to selectively target CSCs.

Clinical Implication of Targeting of Cancer Stem Cells
Emerging evidence has indicated that plenty of special miRNAs are physiologically required for stem cells. For example, miR-124 has shown to regulate both alternative splicing and the transcriptional network promoting neuronal differentiation [66]. Moreover, some miRNAs are responsible for self-renewal and differentiation by negatively regulating the expression of certain key genes in stem cells. In human embryonic stem cells, 8 miRNA loci are located within a 700-bp region on chromosome 4 , while another 4 miRNA loci are mapped within a 1,050-bp region on chromosome 19 [67]. Recently, a study demonstrated that certain miRNAs that regulate the critical promoter of the stem cell self-renewal factor BMI-1 were downregulated in purified populations of normal mammary epithelial stem cells and breast CSCs [64, 68].

The transcriptional repressor zinc-finger E-box binding homeobox 1 (ZEB1) is a crucial inducer of epithelialmesenchymal transition in various human tumors. ZEB1 represses expression of miR-200c, miR-203 and miR-183, which drives the tumor-initiating properties of pancreatic and colorectal cancer cells by upregulating expression of stem cell factors Sox2 and Klf4 [69]. Therefore, the ZEB1-miR-200 feedback loop might form the basis of a promising treatment for solid tumors such as pancreatic cancer [64].

\section{How to Evaluate the Clinical Efficacy of CSC-Targeted Therapy?}

In vitro preclinical models and in vivo xenotransplantations have been initiated developing potential CSC-targeting agents; however, accurate methods evaluating their future efficacy in a clinical setting are still a major challenge. Phase II trials are designed to assess the activity of new compounds using surrogate endpoints to determine whether a phase III trial is warranted [70]. Surrogate markers for measuring CSC activity during and after therapy need to be established. Repetitive quantification of in vitro clonogenic capability as surrogate marker for CSC activity has been successfully correlated in multiple myeloma with progression-free survival and clinical relapse [71]. The commonly accepted criterion for clinical efficacy is overall survival which can only be evaluated in prospective controlled phase III trials requiring large numbers of patients and a long-term follow-up. So far, for acute myeloid leukemia and glioblastoma, a correlation between CSC burden at the time of diagnosis and clinical endpoints such as achievement of 
remission and overall survival has been demonstrated in retrospective analyses [18].

In the future, prospective clinical trials have to be designed to evaluate the clinical efficacy of novel CSC-targeted therapy in combination with critical surrogate markers to demonstrate changes in CSC activity.

\section{Conclusion}

Based on our current knowledge, various properties such as drug resistance as well as migratory and invasive potential are attributed to CSCs in addition to their defining characteristics of self-renewal capacity and tumorigenicity.
Still, the majority of current treatment modalities against cancer target the terminally differentiated cancer cells instead of the CSCs. Currently, there are already some potential anti-CSC agents in preclinical and clinical trials including the notch inhibitors for glioblastoma and the telomerase peptide vaccination after chemoradiotherapy of non-small-cell lung cancer $[58,60]$. While these are promising agents, the likelihood of clinical success will depend on many aspects, including safety, trial design and rational endpoints. To demonstrate the efficacy of CSC-targeted therapy for solid and hematopoietic tumors, further research has to be performed to identify clinically relevant surrogate markers including e.g. frequency, localization and gene signatures of CSCs linking them to clinical endpoints such as overall survival, disease-free survival and relapse-free survival.

\section{References}

1 Gilbert CA, Ross AH: Cancer stem cells: cell culture, markers, and targets for new therapies. J Cell Biochem 2009;108:1031-1038.

-2 Eyler CE, Rich JN: Survival of the fittest: cancer stem cells in therapeutic resistance and angiogenesis. J Clin Oncol 2008;26:28392845.

3 Ghiaur G, et al: Cancer stem cells: relevance to clinical transplantation. Curr Opin Oncol 2012;24:170-175.

4 Dou J, Gu N: Emerging strategies for the identification and targeting of cancer stem cells. Tumour Biol 2010;31:243-253.

5 Vermeulen L, et al: The developing cancer stem-cell model: clinical challenges and opportunities. Lancet Oncol 2012;13:e83-e89.

-6 O'Brien SG, et al: Imatinib compared with interferon and low-dose cytarabine for newly diagnosed chronic-phase chronic myeloid leukemia. N Engl J Med 2003;348:994-1004.

7 Kiladjian JJ, Mesa RA, Hoffman R: The renaissance of interferon therapy for the treatment of myeloid malignancies. Blood 2011; 117:4706-4715.

8 Hochhaus A, et al: Six-year follow-up of patients receiving imatinib for the first-line treatment of chronic myeloid leukemia. Leukemia 2009;23:1054-1061.

-9 Cortes J, O’Brien S, Kantarjian H: Discontinuation of imatinib therapy after achieving a molecular response. Blood 2004;104:22042205.

10 Mauro MJ, Druker BJ, Maziarz RT: Divergent clinical outcome in two CML patients who discontinued imatinib therapy after achieving a molecular remission. Leuk Res 2004;28(suppl 1):S71-S73.

\section{Merante S, et al: Outcome of four patients with chronic myeloid leukemia after ima- tinib mesylate discontinuation. Haemato- logica 2005;90:979-981. \\ 12 Jones RJ, Matsui W: Cancer stem cells: from bench to bedside. Biol Blood Marrow Trans- plant 2007;13(suppl 1):47-52. \\ 13 Jones RJ, Matsui WH, Smith BD: Cancer stem cells: are we missing the target? J Natl Cancer Inst 2004;96:583-585.}

14 Huff CA, et al: The paradox of response and survival in cancer therapeutics. Blood 2006; 107:431-434.

15 Jones RJ: Cancer stem cells - clinical relevance. J Mol Med (Berl) 2009;87:1105-1110.

16 Rasheed ZA, et al: Concise review: emerging concepts in clinical targeting of cancer stem cells. Stem Cells 2011;29:883-887.

17 Garvalov BK, Acker T: Cancer stem cells: a new framework for the design of tumor therapies. J Mol Med (Berl) 2011;89:95-107.

18 Park CY, Tseng D, Weissman IL: Cancer stem cell-directed therapies: recent data from the laboratory and clinic. Mol Ther 2009;17:219-230.

19 Costello R, et al: The immunophenotype of minimally differentiated acute myeloid leukemia (AML-M0): reduced immunogenicity and high frequency of CD34+/CD38- leukemic progenitors. Leukemia 1999;13:15131518.

20 Haferlach T, et al: Morphologic dysplasia in de novo acute myeloid leukemia (AML) is related to unfavorable cytogenetics but has no independent prognostic relevance under the conditions of intensive induction therapy: results of a multiparameter analysis from the German AML Cooperative Group studies. J Clin Oncol 2003;21:256-265.
21 Strojnik T, et al: Neural stem cell markers, nestin and musashi proteins, in the progression of human glioma: correlation of nestin with prognosis of patient survival. Surg Neurol 2007;68:133-143, discussion 143-144.

22 Tu LC, et al: Targeting stem cells - clinical implications for cancer therapy. Curr Stem Cell Res Ther 2009;4:147-153.

23 Klonisch T, et al: Cancer stem cell markers in common cancers - therapeutic implications. Trends Mol Med 2008;14:450-460.

24 Liu R, et al: The prognostic role of a gene signature from tumorigenic breast-cancer cells. N Engl J Med 2007;356:217-226.

25 Tang C, Ang BT, Pervaiz S: Cancer stem cell: target for anti-cancer therapy. FASEB J 2007; 21:3777-3785.

26 Liu G, et al: Analysis of gene expression and chemoresistance of $\mathrm{CD} 133+$ cancer stem cells in glioblastoma. Mol Cancer 2006;5:67.

27 Wright MH, et al: Brcal breast tumors contain distinct $\mathrm{CD} 44+/ \mathrm{CD} 24-$ and CD133+ cells with cancer stem cell characteristics. Breast Cancer Res 2008; 10:R10.

28 Richardson GD, et al: CD133, a novel marker for human prostatic epithelial stem cells. J Cell Sci 2004;117:3539-3545.

29 Collins AT, et al: Prospective identification of tumorigenic prostate cancer stem cells. Cancer Res 2005;65:10946-10951.

-30 Tang M, et al: Dynamics of chronic myeloid leukemia response to long-term targeted therapy reveal treatment effects on leukemic stem cells. Blood 2011;118:1622-1631.

31 Roeder I, et al: Dynamic modeling of imatinib-treated chronic myeloid leukemia: functional insights and clinical implications. Nat Med 2006;12:1181-1184. 
-32 Bardsley MR, et al: Kitlow stem cells cause resistance to Kit/platelet-derived growth factor alpha inhibitors in murine gastrointestinal stromal tumors. Gastroenterology 2010;139:942-952.

- 33 Dylla SJ, et al: Colorectal cancer stem cells are enriched in xenogeneic tumors following chemotherapy. PLoS One 2008;3:e2428.

- 34 Todaro M, et al: Colon cancer stem cells dictate tumor growth and resist cell death by production of interleukin-4. Cell Stem Cell 2007;1:389-402.

-35 Wilson BJ, et al: ABCB5 identifies a therapyrefractory tumor cell population in colorectal cancer patients. Cancer Res 2011;71: 5307-5316.

- 36 Szotek PP, et al: Ovarian cancer side population defines cells with stem cell-like characteristics and mullerian inhibiting substance responsiveness. Proc Natl Acad Sci USA 2006; 103:11154-11159.

- 37 Moserle L, et al: Side population and cancer stem cells: therapeutic implications. Cancer Lett 2010;288:1-9.

38 Fischer S, et al: Identification of multidrug resistance associated proteins MRP1 (ABCC1) and MRP3 (ABCC3) from rainbow trout (Oncorhynchus mykiss). Mar Environ Res 2010;69(suppl):S7-S10.

- 39 Bruns CJ, et al: In vivo selection and characterization of metastatic variants from human pancreatic adenocarcinoma by using orthotopic implantation in nude mice. Neoplasia 1999;1:50-62.

40 Bao S, et al: Glioma stem cells promote radioresistance by preferential activation of the DNA damage response. Nature 2006;444: 756-760.

41 Phillips TM, McBride WH, Pajonk F: The response of CD24(-/low)/CD44+ breast cancer-initiating cells to radiation. J Natl Cancer Inst 2006;98:1777-1785.

42 Diehn M, et al: Association of reactive oxygen species levels and radioresistance in cancer stem cells. Nature 2009;458:780-783.

43 Chen MS, et al: Wnt/beta-catenin mediates radiation resistance of Scal+ progenitors in an immortalized mammary gland cell line. J Cell Sci 2007;120:468-477.

44 Blazek ER, Foutch JL, Maki G: Daoy medulloblastoma cells that express CD133 are radioresistant relative to $\mathrm{CD} 133$ - cells, and the $\mathrm{CD} 133+$ sector is enlarged by hypoxia. Int J Radiat Oncol Biol Phys 2007;67:1-5.

-45 Platet N, et al: Influence of oxygen tension on CD133 phenotype in human glioma cell cultures. Cancer Lett 2007;258:286-290.

$\checkmark 46$ Bentzen SM: Theragnostic imaging for radiation oncology: dose-painting by numbers. Lancet Oncol 2005;6:112-117.
47 Stupp R, et al: Effects of radiotherapy with concomitant and adjuvant temozolomide versus radiotherapy alone on survival in glioblastoma in a randomised phase III study: 5-year analysis of the EORTC-NCIC trial. Lancet Oncol 2009;10:459-466.

48 Mimeault M, et al: Recent advances in cancer stem/progenitor cell research: therapeutic implications for overcoming resistance to the most aggressive cancers. J Cell Mol Med 2007;11:981-1011.

-49 Hosen N, et al: CD96 is a leukemic stem cellspecific marker in human acute myeloid leukemia. Proc Natl Acad Sci USA 2007;104: 11008-11013.

50 Kojima T, Kitamura T: A signal sequence trap based on a constitutively active cytokine receptor. Nat Biotechnol 1999;17:487-490.

51 Lonardo E, et al: Nodal/activin signaling drives self-renewal and tumorigenicity of pancreatic cancer stem cells and provides a target for combined drug therapy. Cell Stem Cell 2011;9:433-446.

-52 Zhou BB, et al: Tumour-initiating cells: challenges and opportunities for anticancer drug discovery. Nat Rev Drug Discov 2009;8:806823.

53 Reya T, et al: Stem cells, cancer, and cancer stem cells. Nature 2001;414:105-111.

54 Reya T, Clevers H: Wnt signalling in stem cells and cancer. Nature 2005;434:843-850.

55 Jiang X, et al: Chronic myeloid leukemia stem cells possess multiple unique features of resistance to $\mathrm{BCR}-\mathrm{ABL}$ targeted therapies. Leukemia 2007;21:926-935

56 Zhao C, et al: Hedgehog signalling is essential for maintenance of cancer stem cells in myeloid leukaemia. Nature 2009;458:776779.

57 Kanamori M, et al: Contribution of Notch signaling activation to human glioblastoma multiforme. J Neurosurg 2007;106:417-427.

58 Jayasekera BA, Bacon AD, Whitfield PC: Management of glioblastoma multiforme in pregnancy. J Neurosurg 2012, E-pub ahead of print.

59 Ju Z, Rudolph KL: Telomeres and telomerase in cancer stem cells. Eur J Cancer 2006:42: 1197-1203.

60 Brunsvig PF, et al: Telomerase peptide vaccination in NSCLC: a phase II trial in stage III patients vaccinated after chemoradiotherapy and an 8-year update on a phase I/II trial. Clin Cancer Res 2011;17:6847-6857.

61 Hurwitz H, et al: Bevacizumab plus irinotecan, fluorouracil, and leucovorin for metastatic colorectal cancer. N Engl J Med 2004 350:2335-2342.
62 Bertout JA, Patel SA, Simon MC: The impact of $\mathrm{O}_{2}$ availability on human cancer. Nat Rev Cancer 2008;8:967-975.

63 Seidel S, et al: A hypoxic niche regulates glioblastoma stem cells through hypoxia inducible factor 2 alpha. Brain 2010;133:983-995.

64 Korpal M, et al: The miR-200 family inhibits epithelial-mesenchymal transition and cancer cell migration by direct targeting of Ecadherin transcriptional repressors ZEB1 and ZEB2. J Biol Chem 2008. 283:1491014914.

65 Liu S, Clouthier SG, Wicha MS: Role of microRNAs in the regulation of breast cancer stem cells. J Mammary Gland Biol Neoplasia 2012;17:15-21.

66 Cao X, Pfaff SL, Gage FH: A functional study of miR-124 in the developing neural tube. Genes Dev 2007;21:531-536.

67 Suh MR, et al: Human embryonic stem cells express a unique set of microRNAs. Dev Biol 2004;270:488-498.

- 68 Xu N, et al: MicroRNA-145 regulates OCT4, SOX2, and KLF4 and represses pluripotency in human embryonic stem cells. Cell 2009; 137:647-658.

-69 Singh A, Settleman J: EMT, cancer stem cells and drug resistance: an emerging axis of evil in the war on cancer. Oncogene 2010;29: 4741-4751.

70 McDermott SP, Wicha MS: Targeting breast cancer stem cells. Mol Oncol 2010;4:404419.

71 Barlogie B, et al: Completion of premaintenance phases in total therapies 2 and $3 \mathrm{im}$ proves clinical outcomes in multiple myeloma: an important variable to be considered in clinical trial designs. Cancer 2008;112: 2720-2725.

$72 \mathrm{Li} \mathrm{C}$, et al: Identification of pancreatic cancer stem cells. Cancer Res 2007;67:1030-1037.

$73 \mathrm{Al}-\mathrm{Hajj} \mathrm{M}$, et al: Prospective identification of tumorigenic breast cancer cells. Proc Natl Acad Sci USA 2003;100:3983-3988.

74 Dalerba P, et al: Phenotypic characterization of human colorectal cancer stem cells. Proc Natl Acad Sci USA 2007;104:10158-10163.

75 Clayton S, Mousa SA: Therapeutics formulated to target cancer stem cells: is it in our future? Cancer Cell Int 2011;11:7.

76 Gibbs CP, et al: Stem-like cells in bone sarcomas: implications for tumorigenesis. Neoplasia 2005;7:967-976.

-77 Yu F, et al: let-7 regulates self renewal and tumorigenicity of breast cancer cells. Cell 2007;131:1109-1123.

-78 Singh SK, et al: REST maintains self-renewal and pluripotency of embryonic stem cells. Nature 2008;453:223-227. 